4 Knabben H, Wolf S, Remky A, Schulte K, Arend O, Reim M. Retinal hemodynamics in patients with hyperviscosity syndrome. Klin. Monatsbl. Augenheilkd. (abstract) 1995; 206(3): 152-156.

5 Dobberstein H, Solbach U, Weinberger A, Wolf S. Correlation between retinal microcirculation and blood viscosity in patients with hyperviscosity syndrome. Clin Hemorheol Microcirc 1999; 20: 31-35.

6 Caimi G. Erythrocyte peroxide metabolism, plasma lipid pattern and hemorheological profile in chronic renal failure. J Nephrol 2002; 15: 104-108.

7 Rezai KA, Patel SC, Eliott D, Becker MA. Rheumatoid hyperviscosity syndrome: reversibility of microvascular abnormalities after treatment. Am J Ophthalmol 2002; 134: 130-132.

8 Saniabadi AR, Fisher TC, Lau CS, Bridges A, Taylor J, Belch J et al. Dipyridamole increases human red blood cell deformability. Eur J Clin Pharmacol 1992; 42: 651-654.

\section{MA Elgohary}

Opthalmology Department

Essex County Hospital, Lexden Road

Colchester $\mathrm{CO} 3$ 3NB, UK

Correspondence: M A Elgohary

Tel: +447939274217

Fax: + 441206744742

E-mail: m.elgohary@doctors.org.uk

Eye (2004) 18, 958-959. doi:10.1038/sj.eye.6701371

Published online 26 March 2004

Sir,

\section{Unusual presentation of dirofilariasis as a lacrimal} mass

A 66-year-old Hong Kong Chinese female patient presented with a drooping of her right upper eyelid for 2 weeks. She was born in China and has never travelled abroad. Examination showed a partial ptosis of her right upper lid. It was caused by a palpable, firm, nontender mass with a size of $1 \mathrm{~cm}$ in diameter located at the superotemporal region of her right orbit. Ophthalmic examination was otherwise normal. Computed tomography scans of the orbit showed an enlarged lacrimal gland of the right eye associated with a nonenhancing hypodense lesion (Figure 1). No adjacent bony erosion was seen. Serological examinations including eosinophil count were normal. Chest X-ray was unremarkable. An excisional biopsy of the lacrimal gland was performed through lateral orbitotomy. The lacrimal gland was found to be well encapsulated and there was no adhesion to the surrounding structures.

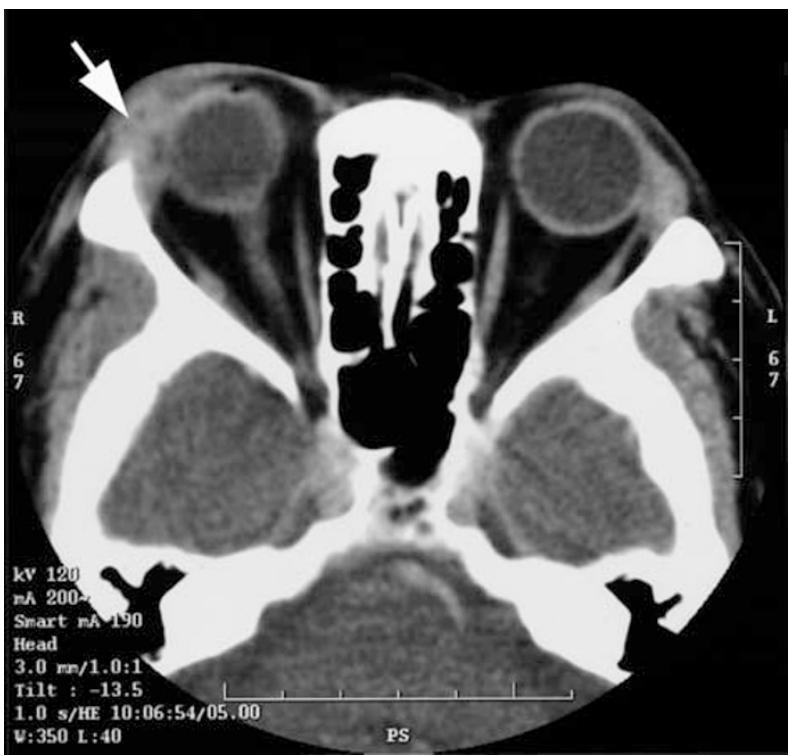

Figure 1 Computed tomographic scan showing an enlarged right lacrimal gland associated with a noncontrast-enhancing hypodense lesion.

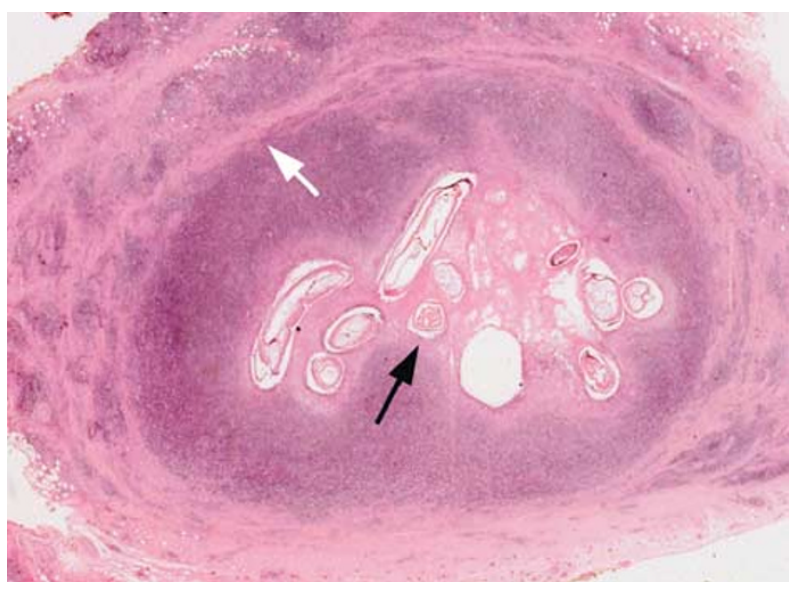

Figure 2 Haematoxylin and eosin stain. Solid arrow: multiple transverse sections of a single female Dirofilaria in the lacrimal gland. Empty arrow: lacrimal gland capsule.

Histopathology demonstrated a parasite surrounded by chronic inflammatory cells and significant eosinophils.

The presence of thick cuticle, numerous external ridges, two internal longitudinal ridges with lateral chords, heavy musculature and double uterine tubes suggested that the parasite was a female Dirofilaria (Figure 2). The degeneration of the specimen did not allow further differentiation into subspecies. After the surgery, the eyelid contour returned to normal and there was no recurrence at the last follow-up visit of 1 year.

Dirofilariasis is a parasitic infection of domestic and wild animals, but it occasionally affects human as zoonotic disease. D. repens is the most frequently 
reported subspecies outside United States. ${ }^{1}$ Mosquitoes are the biological vectors in transmitting the parasite from animals to human. Sometimes they can penetrate the tissue and spread systemically into internal organs. ${ }^{2}$ Pampiglione $e t \mathrm{al}^{3}$ reported a case series of dirofilarial infections in which $30 \%$ had ocular involvement. Ocular infection can be subconjunctival, periorbital, orbital, or intraocular. Orbital infections can result in proptosis, ptosis, or diplopia. ${ }^{4}$

Systemic features such as fever, lymphadenopathy, and reactive arthritis are occasionally seen. Only 25\% of subcutaneous cases has peripheral eosinophilia. ${ }^{4}$ The diagnosis is made on the basis of histological and morphological identification. Serology and molecular biology techniques may provide supportive information, but the significance of them being the definitive diagnostic tool has not yet been established. ${ }^{3,5}$

In our case, the acquisition of the parasite may have occurred in Hong Kong or in China. Neither the time of transmission nor the route of entry into the lacrimal gland can be determined. Direct inoculation into lacrimal gland is probably not possible. One possibility is that, following an innocuous mosquito bite in the periorbital region, the microfilaria developed in the subcutaneous tissue and finally burrowed into the lacrimal gland. Surgical excision is the recommended treatment for dirofilariasis and this can also provide histological diagnosis. Unlike other filarial infections such as onchocerciasis and loiasis, microfilaraemia is unusual and antihelminthic agents are generally not indicated.

To the best of our knowledge, this is the only report in the English literature that dirofilariasis presented as a lacrimal mass. The initial clinical and radiological findings were all suggestive of a benign tumour. The deceptive feature led to a diagnostic challenge. One should consider this as an unusual differential diagnosis especially for those who have travelled in the past to endemic areas.

\section{Acknowledgements}

Financial support: Nil; proprietary interest: Nil.

\section{References}

1 Ruiz-Moreno JM, Bornay-Llinares FJ, Maza GP, Medrano M, Simon F, Eberhard ML. Subconjunctival infection with dirofilaria repens. Arch Ophthalmol 1998; 116: 1370-1372.

2 Settnes OP, Engebjerb E. Human subcutaneous dirofilariasis caused by Dirofilariarepens: report of a case in Norway and review of recent literature. Acta Pathol Microbiol Immunol Scand 1991; 99: 364-370.

3 Pampiglione S, Rivasi F, Boldorini R, Incensati RM, Pastormerlo M, Pavesi M et al. Dirofilariasis due to Dirofilaria repens in Italy, an emergent zoonosis: report of 60 new cases. Histopathology 2001; 38: 344-354.

4 Johnson TM, Gilberg S, Robertson S. Unusual presentation of periorbital dirofilariasis. Can J Ophthalmol 1998; 33: 333-335.

5 Arvanitis PG, Vakalis NC, Damanakis AG, Theodossiadis GP. Ophthalmic dirofilariasis. Am J Ophthalmol 1997; 123: 689-690.

KSC Yuen'1, MWI Tse', PCL Choi², W-M Chan' and DSC Lam $^{1}$

'Department of Ophthalmology and Visual

Sciences, The Chinese University of Hong Kong

Prince of Wales Hospital

Shatin, N.T., Hong Kong

${ }^{2}$ Department of Anatomical and Cellular Pathology, The Chinese University of Hong Kong

Prince of Wales Hospital

Shatin, N.T., Hong Kong

Correspondence: KSC Yuen

Tel: +85226322878

Fax: + 85226482943

E-mail: kenneth-yuen@mail.hongkong.com

Eye (2004) 18, 959-960. doi:10.1038/sj.eye.6701372

Published online 19 March 2004

Sir,

Giant retinal pigment epithelium rip secondary to subretinal proliferative vitreoretinopathy

Retinal pigment epithelial (RPE) rips occur relatively uncommonly and are usually associated with serous detachments of the pigment epithelium, secondary to choroidal neovascularization. These typically occur at the macular and cause rapid visual loss. Once the pigment epithelial tear occurs, the RPE retracts from the outer portion of Bruch's membrane and scrolls up. Fluorescein angiography typically demonstrates a well-demarcated hyperfluorescence in the early phase of the angiogram. We describe a case of RPE tear, unusual in terms of its pathogenesis and size.

\section{Case report}

An 81-year-old lady was referred with deteriorating vision in her right eye over a period of weeks. Her left eye was blind due to an inoperable retinal detachment 60 years previously. Examination revealed acuity of 6/9 in her right eye and hand movements in her left. On fundoscopy, the left eye had a chronic inferior macular off retinal detachment. Above the superior arcade, there 